\title{
Long-term outcomes of surgery and radiotherapy for secreting and non-secreting pituitary adenoma
}

\author{
Mi Young Kim, MD¹, Jin Hee Kim, MD, PhD¹, Young Kee Oh, PhD'1, El Kim, MD, PhD² \\ Departments of ${ }^{1}$ Radiation Oncology and ${ }^{2}$ Neurosurgery, Dongsan Medical Center, \\ Keimyung University School of Medicine, Daegu, Korea
}

\begin{abstract}
Purpose: To investigate treatment outcome and long term complication after surgery and radiotherapy (RT) for pituitary adenoma.

Materials and Methods: From 1990 to 2009, 73 patients with surgery and RT for pituitary adenoma were analyzed in this study. Median age was 51 years (range, 25 to 71 years). Median tumor size was $3 \mathrm{~cm}$ (range, 1 to $5 \mathrm{~cm}$ ) with suprasellar $(n=21)$, cavernous sinus extension $(n=14)$ or both $(n=5)$. Hormone secreting tumor was diagnosed in 29 patients; 16 patients with prolactin, 12 patients with growth hormone, and 1 patient with adrenocorticotrophic hormone. Impairment of visual acuity or visual field was presented in 33 patients at first diagnosis. Most patients $(n=64)$ received RT as postoperative adjuvant setting. Median RT dose was 45 Gy (range, 45 to 59.4 Gy).

Results: Median follow-up duration was 8 years (range, 3 to 22 years). In secreting tumors, hormone normalization rate was 55\% (16 of 29 patients). For 25 patients with evaluable visual field and visual acuity test, 21 patients (84\%) showed improvement of visual disturbance after treatment. The 10-year tumor control rate for non-secreting and secreting adenoma was 100\% and 58\%, respectively $(p<0.001)$. Progression free survival rate at 10 years was $98 \%$. Only 1 patient experienced endocrinological recurrence. Following surgery, $60 \%(n=44)$ suffered from pituitary function deficit. Late complication associated with RT was only 1 patient, who developed cataract.

Conclusion: Surgery and RT are very effective and safe in hormonal and tumor growth control for secreting and non-secreting pituitary adenoma.
\end{abstract}

Keywords: Pituitary adenoma, Radiotherapy, Treatment outcome, Complication

\section{Introduction}

Pituitary adenoma is a benign tumor representing 10\% to $15 \%$ of primary intracranial tumors. This tumor may cause nonspecific headache, diminished vision, diplopia, hypopituitarism, and other neurologic symptoms as a result of mass effect on adjacent intracranial structures. Functioning adenoma may cause various metabolic symptoms due to hypersecretion of pituitary hormone, such as prolactin (PRL), growth hormone (GH), thyroid-stimulating hormone, adrenocorticotropic hormone (ACTH), and gonadotropins. In patients with non-secreting macroadenoma or non-prolactinsecreting adenoma, surgery (usually the transsphenoidal approach) is considered the treatment of choice. In prolactin-

Received 18 February 2016, Revised 14 March 2016, Accepted 31 March 2016.

Correspondence: Jin Hee Kim, MD, PhD, Department of Radiation Oncology, Dongsan Medical Center, Keimyung University School of Medicine, 56 Dalseong-ro, Jung-gu, Daegu 41931, Korea. Tel: +82-53-250-7665, Fax: +82-53-250-7487, E-mail: jhkim@dsmc.or.kr

(C) This is an Open Access article distributed under the terms of the Creative Commons Attribution Non-Commercial License (http://creativecommons.org/ licenses/by-nc/4.0/) which permits unrestricted non-commercial use, distribution, and reproduction in any medium, provided the original work is properly cited.

www.e-roj.org 
secreting adenoma, medical treatment is the treatment of choice. Radiotherapy (RT) usually is applied to patients with residual or recurrent disease after surgical resection, patients who are not operative candidates, and patients with secreting adenoma that do not respond to surgery or medication. RT can achieve excellent long term tumor control, with reported rates of 80\%-95\% at 10 years after $45-50$ Gy [1-4].

Nevertheless, RT has been associated with possible longterm complications, such as hypopituitarism, cerebrovascular accident, damage to the visual apparatus, and secondary brain neoplasm. The incidence of hypopituitarism has been reported to be $20 \%-60 \%$ at $5-10$ years after RT $[1,2,4-6]$. Some authors reported that cerebrovascular accident (usually ischemic stroke) was increased after RT $[5,7]$. However, it is unclear that RT is associated with increased incidence of these complication, and its cause is likely multifactorial. In case of stroke, other factors, such as diabetes mellitus, heart arrhythmia, can play an important role to develop stroke.

Pituitary adenoma is a benign tumor that has a long natural history, and complication can occur even more than 10 years after RT, so proper evaluation of treatment requires a long-term follow-up $[2,4,5,7]$. This study was performed to investigate the long-term outcome and complication after surgery and RT for pituitary adenoma.

\section{Materials and Methods}

\section{Patient characteristics}

From 1999 to 2009, 101 patients who had diagnosed pituitary adenoma received RT in Dongsan Medical Center, Keimyung University School of Medicine. Of these patients, 21 patients without regular follow-up, 4 patients without medical records, 3 patients with incomplete RT were excluded, and 73 patients were analyzed in this study. Patient characteristics are summarized in Table 1. Median age was 51 years (range, 25 to 71 years). Median tumor size was $3 \mathrm{~cm}$ (range, 1 to $5 \mathrm{~cm}$ ) with suprasellar $(n=21)$, cavernous sinus extension $(n=14)$ or both $(n=5)$. Forty-four patients $(60 \%)$ had non-secreting adenomas, and $29(40 \%)$ had secreting adenomas. For secreting adenomas, patients, who do not respond to hormone suppressive medication or first operation, received RT. Massrelated symptoms comprised; 28 patients with visual field defect or visual acuity impairment, 18 patients with headache, and 3 patients with hypopituitarism. Four patients had both visual field defect and headache, and 1 patient had both visual field defect and hypopituitarism. Regarding the 29 secreting adenomas, 16 were PRL secreting, 12 were GH secreting, and
Table 1. Patients and tumor characteristics

\begin{tabular}{lc}
\hline \multicolumn{1}{c}{ Variable } & No. of patients (\%) \\
\hline Age (yr), median (range) & $51(25-71)$ \\
Gender & $33(45)$ \\
Male & $40(55)$ \\
Female & $3(1-5)$ \\
Tumor size (cm), median (range) & \\
Tumor extension & $18(25)$ \\
Sella only (no extension) & $26^{\text {a) }}(36)$ \\
Suprasellar & $19^{\text {a) }}(26)$ \\
Cavernous sinus & $2(3)$ \\
Sphenoid sinus & $13(18)$ \\
Unknown & \\
Hormone secreting & $44(60)$ \\
Non-secreting & $29(40)$ \\
Secreting & 16 \\
Prolactin & 12 \\
Growth hormone & 1 \\
Adrenocorticotrophic hormone & \\
\hline
\end{tabular}

${ }^{a} 5$ patients had both suprasella and cavernous sinus extension.

1 was ACTH secreting. Symptoms of secreting adenomas were present in 27 patients (2 patients with elevated PRL had no hormone-related symptoms). Acromegaly ( $n=12)$, amenorrhea $(n=11)$, hypogonadism $(n=2)$, Cushing's disease $(n=1)$, and gynecomastia $(n=1)$ were present before surgery.

\section{Treatment}

In this analysis, all patients received surgery before RT. Sixty four patients had RT in the postoperative adjuvant setting. In the remaining 9 patients, RT was performed as a salvage treatment for recurrent or persistent disease after the first operation. Most patients $(n=70)$ had surgery with transsphenoidal approach. Surgical extent was determined by imaging methods like computed tomography (CT) or magnetic resonance imaging (MRI), operation record, or surgeon's opinion. Based on brain CT or MRI after operation, gross total resection was defined as no residual tumor, and subtotal resection was defined as residual tumor enhancement. Of total 73 patients, 64 patients who had subtotal resection of tumor received RT as postoperative adjuvant setting. For RT, CT simulation was performed in patients immobilized using a thermoplastic mask. Before three-dimensional (3D) conformal therapy, simulation film was used to shield and shape the radiation field, and treated with the 3 -field technique (1 antero-superior oblique and 2 lateral fields). In 3D conformal therapy, gross tumor volume (GTV) was defined as gross disease, which was seen in the contrast-enhanced CT or MRI. 
Median tumor dose was 45 Gy (range, 45 to 59.4 Gy) with 1.8 Gy per fraction, once a day, 5 times a week.

Two patients with PRL secreting adenoma, 1 patient with ACTH secreting, and 1 patient with $\mathrm{GH}$ secreting treated with medication, such as bromocriptine, ketoconazole, and octreotide either before or after surgery.

\section{Evaluation of treatment results}

The follow-up period was calculated from the last date of RT to time of last follow-up or death. Treatment outcomes was evaluated by ophthalmological (visual acuity and visual field test), endocrinological (thyroid stimulating hormone, thyroxine, triiodothyronine, $\mathrm{ACTH}, \mathrm{PRL}, \mathrm{GH}$, luteinizing hormone, and follicle-stimulating hormone) and radiological (brain MRI) studies after completion of RT at 6 months, 1 year and then once a year.

The primary outcome of this study were tumor control, disease-specific survival (DSS), overall survival (OS), progression-free survival (PFS) and hormone normalization rate of secreting adenomas. In non-secreting adenoma, tumor control was defined as lack of radiological progression on follow-up brain imaging studies and lack of mass-related symptom. Regardless of conservative management, if there was an improvement of mass-related symptom, such as headache, it was defined as resolution of symptom. In secreting adenoma, tumor control was defined as normalization of hypersecreted hormones, maintenance of normalized status, and lack of radiological progression. Secreting adenomas was considered to have had a hormone normalization to RT if there hypersecreted hormone decreased to the normal range without suppressive medication after surgery and RT. Progression free interval was defined as duration from the date of end of RT to the date of radiological or endocrinological relapse after control of tumor. The tumor control rate DSS, OS, and PFS rate was calculated using Kaplan-Meier method. For patients who never achieved hormone normalization during the follow-up period, we considered that patients did not have tumor control at the time of last follow-up time.

The secondary outcome of interest in this study was treatment-related chronic complication, including hypopituitarism, radiation-induced optic neuropathy, cataract, cerebrovascular accident, and so on.

\section{Results}

Median follow-up duration was 8 years (range, 3 to 22 years) and 26 patients (36\%) had 10 year or more follow-up duration.
RT was well tolerated by all patients. No acute toxicities during RT was observed except headache reported by 16 patients (all grade 1), and nausea reported by 3 patients (all grade 1 or 2 ).

\section{Treatment outcomes}

Of the 29 patients with all type of secreting adenomas, with a median follow-up duration of 8 years, 16 patients (55\%) showed complete hormone normalization without suppressive medication. The 10 -year tumor control rate for secreting adenoma was 58\% (Fig. 1). The median time to hormone normalization after RT was 3.1 years (range, 0.1 to 10.1 years). Of the 16 patients with PRL secreting adenoma, 7 patients (44\%) had hormone normalization after median 2.4 years. Of the 12 patients with $\mathrm{GH}$ secreting adenoma, 9 patients (75\%) had hormone normalization after a median 4.9 years. One patient with ACTH secreting adenoma still required suppressive medication, because that patient never achieved hormone normalization. There was no difference between radiation dose and hormone normalization rate.

Of the 23 patients with headache, all patients showed resolution of symptoms after surgery and RT. Of the 33 patients with initial visual field defect or visual acuity impairment, 8 patients did not have ophthalmologic test during the follow-up period. For 25 patients with evaluable visual field and visual acuity test, 21 patients (84\%) showed improvement of visual disturbance after treatment (17 patients after surgery and 4 patients after RT).

The 10-year tumor control rate for non-secreting and secreting adenoma was $100 \%$ and 58\%, respectively ( $p<$ 0.001). Whether the adenoma was secreting or non-secreting was the significant prognostic factor for local control in multivariate analysis ( $p=0.015)$ (Table 2). Tumor extension,

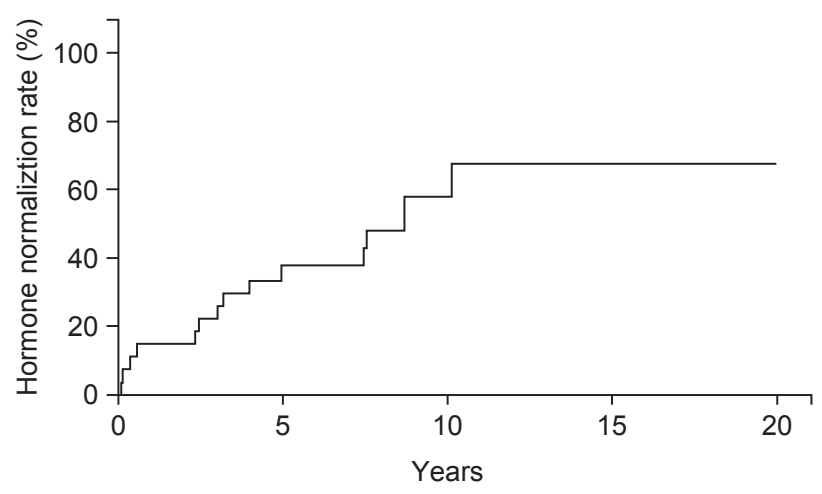

Fig. 1. Hormone normalization rate after treatment. Sixteen patients (55\%) showed complete hormone normalization with median follow-up duration of 8 years. 
Table 2. Multivariate analysis of factors related to tumor control

\begin{tabular}{lcc}
\hline \multicolumn{1}{c}{ Variable } & $\mathrm{HR}(95 \% \mathrm{Cl})$ & $\mathrm{p}$-value \\
\hline Secreting status & $2.491(1.195-5.193)$ & $0.015^{*}$ \\
Radiation dose & - & 0.299 \\
Tumor extension & - & 0.339 \\
Tumor size & - & 0.345 \\
\hline
\end{tabular}

$\mathrm{HR}$, hazard ratio; $\mathrm{Cl}$, confidence interval.

${ }^{*} p<0.05$.

radiation dose, and tumor size did not affect the tumor control.

The DSS and OS rate at 10 years was 100\% and 92.8\%, respectively. Of the 5 patients who died during the followup period, none had a cause of death that was attributable to the pituitary adenoma, and RT; traffic accident, gastric cancer, chronic renal failure, pontine infarction, and unknown cause. Patients who died due to pontine infarction had uncontrolled diabetes mellitus. Thus, the pituitary adenoma itself or treatment might be not associated with death (see below "treatment-related late complication"). The PFS rate at 10 years was 98\%. There was no tumor regrowth of nonsecreting adenoma on follow-up brain image. However, 1 patient experienced endocrinological recurrence of disease 4.5 years after RT leading to hormone suppressive medication. This patient required octreotide to inhibit secretion and action from $\mathrm{GH}$ until last follow-up.

\section{Treatment-related late complication}

After surgery, 44 patients (60\%) suffered from pituitary function deficit, and had hormone replacement with levothyroxine, and/or hydrocortisone. After RT, none had newly diagnosed as treatment-related hypopituitarism.
Headache was the most common acute complication during RT ( $n=13$ ), and all was the grade I (Common Terminology Criteria for Adverse Events [CTCAE] ver. 4). Of 13 patients, 10 patients had headache before surgery. These 10 patients with preoperative headache were defined as tumor control, not progression of tumor, because preoperative headache was disappeared after surgery, and grade I headache during RT was also disappeared after RT despite of no conservative management.

Six patients (8.2\%) experienced ischemic stroke after a median 5.3 years (range, 2.3 to 8.5 years) (Table 3 ). Although stroke of pons and midbrain were close or in the RT field, it is unclear that RT was the causative factor of ischemic stroke, because 2 patients (patients 1,2) with pons infarction had uncontrolled diabetes mellitus, which is strong risk factor of ischemic stroke. Patients 5 and 6 had heart arrhythmia (atrial fibrillation) as a cause of stroke, and stroke lesions were far from the radiation field.

One patient who had received RT at 66 years experienced cataract after 7 years. No patient experienced radiationinduced optic neuropathy, brain necrosis, and secondary malignancy as a result of RT.

\section{Discussion and Conclusion}

This study is a retrospective analysis with long-term followup results of surgery and RT for pituitary adenoma. Our study confirms that surgery and RT are very effective and safe in hormonal and tumor growth control, similarly, in other studies. Numerous studies demonstrated that surgery followed by RT or RT alone achieved excellent long-term outcome of both secreting and non-secreting pituitary adenoma. Brada et al.

Table 3. Cerebrovascular accident after treatment

\begin{tabular}{|c|c|c|c|c|c|c|c|c|}
\hline Patient & Age $(y r)^{a)}$ & Sex & Tumor type & $\begin{array}{l}\text { RT dose } \\
\text { (cGy) }\end{array}$ & $\begin{array}{c}\text { Time since } \\
\text { RT (yr) }\end{array}$ & $\begin{array}{c}\text { Hormone } \\
\text { normalization }\end{array}$ & Stroke lesion & $\begin{array}{l}\text { Stroke risk } \\
\text { factors }\end{array}$ \\
\hline 1 & 53 & $M$ & Secreting $(\mathrm{GH})$ & 5,400 & 5.2 & Yes & Pons & DM \\
\hline 2 & 29 & $\mathrm{~F}$ & Secreting (PRL) & 5,400 & 3.8 & Yes & Pons & DM \\
\hline 3 & 68 & $\mathrm{~F}$ & Non-secreting & 5,400 & 8.5 & - & Midbrain & - \\
\hline 4 & 59 & M & Non-secreting & 5,220 & 2.3 & - & Unknown & HTN \\
\hline 5 & 66 & M & Non-secreting & 4,500 & 6.0 & - & $\begin{array}{l}\text { Right inferior } \\
\text { cerebellum }\end{array}$ & Arrhythmia \\
\hline 6 & 48 & $\mathrm{~F}$ & Non-secreting & 5,400 & 5.5 & - & $\begin{array}{l}\text { Left frontal lobe } \\
\text { (subcortical area) }\end{array}$ & Arrhythmia \\
\hline
\end{tabular}

RT, radiotherapy; $M$, male; F, female; $G H$, growth hormone; DM, diabetes mellitus; $P R L$, prolactin; HTN, hypertension; Arrhythmia, atrial fibrillation.

${ }^{a}$ Age at radiotherapy treatment. 
[1] reported the results of 411 patients including secreting and non-secreting adenomas. The actuarial PFS was 94\% at 10 years and $88 \%$ at 20 years for all patients. Only secretory status was an independent prognostic factor. According to the study of Snead et al. [2], the 10-year actuarial local control rates for non-secreting and secreting adenomas were $98 \%$ and $73 \%$, respectively ( $p=0.0015)$. In this study, the presence of hormone-secreting tumors was the only prognostic factor that significantly influenced outcome. Sasaki et al. [3] reported that conventional RT with 50 Gy was safe and sufficient to control pituitary adenoma. The 10-year local control rates was $98 \%$ and $83 \%$ for non-secreting and all type of secreting adenoma $(p=0.006)$. In our study, the 10 -year tumor control rates for non-secreting and secreting adenoma was 100\% and 58\%, respectively $(p<0.001)$. To improve tumor control of secreting adenoma, multimodality approaches including, surgery, medical treatment, and RT are needed. In case of RT, higher radiation dose to tumor without increased complication is needed. The modern RT technique of, stereotactic radiosurgery (SRS) provides advantages over external beam radiotherapy (EBRT) including patient's convenience (single session treatment) and better ability to spare normal pituitary and neural structures. SRS is an also very effective treatment modality for pituitary tumor, with tumor control rate of around 90\% [8]. However, it is important to note that EBRT has been used to treat pituitary adenoma for a longer follow-up than reported in the SRS literature. So, the SRS literature has to become mature, with a longer follow-up period.

For all type of secreting adenomas, 55\% presently showed complete hormone normalization. Rate of hormone normalization of PRL and GH secreting adenoma was $44 \%$ and $75 \%$, respectively, which is comparable to previous reports. In previous reports, rates of normalization of serum PRL levels in prolactinoma ranged from $12 \%$ to $60 \%$, and rates of normalization serum of $\mathrm{GH}$ levels in patients with $\mathrm{GH}$ secreting adenoma ranged from $61 \%$ to $77 \%$ [9-12]. Some studies reported that rates of hormone normalization were increased with increased follow-up duration. Jenkins et al. [11] reported on 884 patients who received conventional radiation for pituitary GH secreting adenoma, and described that $22 \%$ of patients achieved a GH level $\leq 2.5 \mathrm{ng} / \mathrm{mL}$ by 2 years, $60 \%$ by 10 years, and $77 \%$ by 20 years. Minniti et al. [12] reported normal age-corrected insulin-like growth factor (IGF)-I levels in $8 \%$ of patients 2 years after RT, and this proportion increased to $23 \%, 42 \%$, and $61 \%$ after 5,10 , and 15 years, respectively. Our results also showed an increase in the rate of hormone normalization with increasing follow-up duration until 10 years after RT (Fig. 1). Therefore, to evaluate the efficacy of RT for secreting adenoma, long-term follow-up, more than 10 years is needed.

In the present study, the median time to hormone normalization after RT was 3.1 years (range, 0.1 to 10.1 years). Some authors have suggested that SRS achieved faster control of hypersecreted hormones than does conventional fractionated RT $[8,13]$. However, these suggestions should be interpreted cautiously, because tumor characteristics are different between SRS and conventional fractionated RT. SRS is a technique of stereotactically guided high dose conformal irradiation to target volume in single fraction. Therefore, there are some requirements for successful SRS (while minimizing radiation dose to surrounding normal tissue) as follows; small target volume, sharply defined target, accurate radiation delivery, and dose-limiting structures excluded from target. So, mostly, tumor characteristics of SRS literatures are smaller tumor volume, no diffuse infiltration, and no close proximity to optic apparatus, compared with conventional fractionated RT literatures.

Hypopituitarism is most common late complication after RT for pituitary adenoma. Its incidence is varies from 20\% to 60\%, depending on the duration of follow-up [1,2,4-6]. However, its cause is likely multifactorial; RT to the normal pituitary gland, surgical manipulation, or functional disruption of the pituitary by the adenoma itself. In our hospital, 44 patients experienced new-hypopituitarism after surgery, and these patients had hormone substitution. After RT, there was no patient who was newly diagnosed as hypopituitarism. However, this result should be interpreted cautiously. In our institution, patients received levothyroxine and hydrocortisone after surgery, according to pituitary hormonal study or extent of surgical procedure. For patients who were taking levothyroxine and hydrocortisone according to the decision of neurosurgeon, even if hormone levels were normal after surgery, there has been the possibility of masking of RT-associated hypopituitarism. Another study described that immediate postoperative RT (group 1) did not result in an additional need for conventional hormonal substitution treatment, compared with surgery and a 'wait and see policy' (group 2) [14]. There were no differences in hormone substitution free survival with respect to thyroid hormone, glucocorticoids, and sex hormones between the 2 groups. The study authors suggested that immediate postoperative RT provide excellent local control without an additional deleterious effect. Although many studies including conventional fractionated RT, and SRS reported hypopituitarism as most common complication after 
RT, surgical manipulation to the hypothalamus-pituitary axis may be an important causative factor of hypopituitarism. In addition to surgery, longer follow-up may be also associated with increased incidence of hypopituitarism. In a study of Hoybye et al. [15], hypopituitarism after SRS occurred even more than 10 years after treatment. Therefore, long-term, regular hormonal study is required after surgery and RT.

Stroke is also important late complication after RT. Some investigators reported on increased incidence of stroke in patients treated with RT compared with normal population. Brada et al. [7] reported a relative risk (RR) of stroke of 4.1, and Erridge et al. [5] reported a RR of 1.45 for men, 2.22 for women. Both studies had a median follow-up time of 15 years and 9 years, respectively. However, in a recent study of Sattler et al. [16], postoperative RT was not associated with an increased incidence of stroke compared with surgery alone. There was also no difference in lesions of stroke or causative mechanism based on stroke subtype classification system. They suggested that risk factors other than RT may have contributed to the development of stroke. Risk factors included pre-existent coronary or peripheral artery disease, surgical trauma to the vasculature, hormone excess, hormone deficiency, hypertension, dyslipidemia, and diabetes mellitus. In our studies, 6 patients (8\% of total patients) experienced cerebrovascular accident (ischemic stroke) after surgery and RT. The median time to development of ischemic stroke after treatment was 5.35 years (range, 2.3 to 6.0 years). However, it is unclear that whether the RT is associated with ischemic stroke, because many risk factors can contribute the development of ischemic stroke. The lesion of stroke for 2 patients with pontine infarction was close or within RT field, and RT dose was $54 \mathrm{~Gy}$. However, these 2 patients had diabetes mellitus as a risk factor of ischemic stroke. We cannot exclude the possibility that surgery and RT contributed the development of ischemic stroke for these 2 patients. Two other patients had heart arrhythmia as stroke risk factors and lesions of stroke were far from RT field.

There was no second brain malignancy during the followup period. This is very important because some physicians may be hesitant to conduct RT for benign neoplasm in critical locations. Minniti et al. [17] reported that 11 of 426 patients developed a second brain tumor, including 5 meningiomas, 4 high grade astrocytomas, 1 meningeal sarcoma, and 1 primitive neuroectodermal tumor. The cumulative risk was $2.4 \%$ at 20 years, with relative risk of 10.5 , compared with normal population. Erridge et al. [5] reported that the 20-year actuarial risk of a second brain tumor was $1.9 \%$, with relative risk of 5.65 (for men) and 9.94 (for women). However, how much of this excess risk is attributed to the RT, and whether or not having pituitary adenoma itself increases the risk is not known. Moreover, the risk of second brain tumor of 20 years remains low. This low incidence should not preclude the use of RT as an effective treatment modality for pituitary adenoma.

In conclusion, with median 8 years follow-up, surgery and RT are very effective and safe in hormonal and tumor growth control for secreting and non-secreting pituitary adenoma. However, efforts are needed to improve hormonal control for secreting adenoma.

\section{Conflict of Interest}

No potential conflict of interest relevant to this article was reported.

\section{References}

1. Brada M, Rajan B, Traish D, et al. The long-term efficacy of conservative surgery and radiotherapy in the control of pituitary adenomas. Clin Endocrinol (Oxf) 1993;38:571-8.

2. Snead FE, Amdur RJ, Morris CG, Mendenhall WM. Long-term outcomes of radiotherapy for pituitary adenomas. Int J Radiat Oncol Biol Phys 2008;71:994-8.

3. Sasaki R, Murakami M, Okamoto $Y$, et al. The efficacy of conventional radiation therapy in the management of pituitary adenoma. Int J Radiat Oncol Biol Phys 2000;47:1337-45.

4. Tsang RW, Brierley JD, Panzarella T, Gospodarowicz MK, Sutcliffe SB, Simpson WJ. Radiation therapy for pituitary adenoma: treatment outcome and prognostic factors. Int $J$ Radiat Oncol Biol Phys 1994;30:557-65.

5. Erridge SC, Conkey DS, Stockton D, et al. Radiotherapy for pituitary adenomas: long-term efficacy and toxicity. Radiother Oncol 2009;93:597-601.

6. Rim CH, Yang DS, Park YJ, Yoon WS, Lee JA, Kim CY. Radiotherapy for pituitary adenomas: long-term outcome and complications. Radiat Oncol J 2011;29:156-63.

7. Brada M, Burchell L, Ashley S, Traish D. The incidence of cerebrovascular accidents in patients with pituitary adenoma. Int J Radiat Oncol Biol Phys 1999;45:693-8.

8. Ding D, Starke RM, Sheehan JP. Treatment paradigms for pituitary adenomas: defining the roles of radiosurgery and radiation therapy. J Neurooncol 2014;117:445-57.

9. Sheplan Olsen $\sqcup$, Robles Irizarry L, Chao ST, et al. Radiotherapy for prolactin-secreting pituitary tumors. Pituitary 2012;15:135-45.

10. Barrande G, Pittino-Lungo $M$, Coste J, et al. Hormonal and metabolic effects of radiotherapy in acromegaly: long-term 
results in 128 patients followed in a single center. J Clin Endocrinol Metab 2000;85:3779-85.

11. Jenkins PJ, Bates P, Carson MN, Stewart PM, Wass JA. Conventional pituitary irradiation is effective in lowering serum growth hormone and insulin-like growth factor-I in patients with acromegaly. J Clin Endocrinol Metab 2006;91:1239-45.

12. Minniti $G$, Jaffrain-Rea $M L$, Osti $M$, et al. The long-term efficacy of conventional radiotherapy in patients with $\mathrm{GH}$-secreting pituitary adenomas. Clin Endocrinol (Oxf) 2005;62:210-6.

13. Loeffler JS, Shih HA. Radiation therapy in the management of pituitary adenomas. J Clin Endocrinol Metab 2011;96:19922003.

14. van den Bergh AC, van den Berg G, Schoorl MA, et al. Immediate postoperative radiotherapy in residual nonfunctioning pituitary adenoma: beneficial effect on local control without additional negative impact on pituitary function and life expectancy. Int J Radiat Oncol Biol Phys 2007;67:863-9.

15. Hoybye C, Grenback E, Rahn T, Degerblad M, Thoren M, Hulting AL. Adrenocorticotropic hormone-producing pituitary tumors: 12- to 22-year follow-up after treatment with stereotactic radiosurgery. Neurosurgery 2001;49:284-91.

16. Sattler MG, Vroomen PC, Sluiter WJ, et al. Incidence, causative mechanisms, and anatomic localization of stroke in pituitary adenoma patients treated with postoperative radiation therapy versus surgery alone. Int J Radiat Oncol Biol Phys 2013;87:53-9.

17. Minniti G, Traish D, Ashley S, Gonsalves A, Brada M. Risk of second brain tumor after conservative surgery and radiotherapy for pituitary adenoma: update after an additional 10 years. J Clin Endocrinol Metab 2005;90:800-4. 